Dalibor Pražák, Kumbakonam R. Rajagopal

\title{
Mechanical oscillators described by a system of differential-algebraic equations
}

Preprint No. MATH-KMA-2009/316

December 2009

Submitted

Department of Mathematical Analysis, Faculty of Mathematics and Physics, Charles University, Sokolovská 83, 18675 Praha 8, Czech Republic

http://www.karlin.mff.cuni.cz/kma-preprints/ 
Cover print by Reprostředisko, Malostranské nám. 25, Praha 1 - Malá Strana Design by Mirko Rokyta, picture of snowflake by Ondřej Kalenda

Current series administrator: Mirko Rokyta 


\section{List of preprints released in this series}

All preprints available on http://www.karlin.mff.cuni.cz/kma-preprints/ E-mail contact to series administrator: kma-prep@karlin.mff.cuni.cz 
For the details on the 255 papers submitted in years 1999-2007 see the web page of the preprint series.

\section{8}

[MATH-KMA 2008/256] Miroslav Bulíček, Dalibor Pražák: A note on the dimension of the global attractor for abstract semilinear hyperbolic problem.

[MATH-KMA 2008/257] Eva Fašangová, Pedro J. Miana: Hilbert, Dirichlet and Fejér families of operators arising from $C_{0}$-groups and cosine functions.

[MATH-KMA 2008/258] Josef Daněček, Oldřich John, Jana Stará: On the Hölder continuity of gradient of solutions to a class of elliptic systems.

[MATH-KMA 2008/259] Jakub Duda, Luděk Zajíček: Semiconvex functions: representations as suprema of smooth functions and extensions.

[MATH-KMA 2008/260] Jakub Duda, Luděk Zajíček: Curves in Banach spaces which allow a $C^{2}$ parametrization.

[MATH-KMA 2008/261] Václav Vlasák, Miroslav Zelený: Compact sets of continuity for Borel functions.

[MATH-KMA 2008/262] Ondřej Kalenda, Jiří Spurný: Boundaries of compact convex sets and fragmentability.

[MATH-KMA 2008/263] Ondřej Kurka, Dušan Pokorný: Porosity and the Darboux property of Fréchet derivatives.

[MATH-KMA 2008/264] Miroslav Kačena, Jiří Spurný: Affine images of compact convex sets and maximal measures.

[MATH-KMA 2008/265] Miroslav Kačena: Products and projective limits of function spaces.

[MATH-KMA 2008/266] Miroslav Bačák: Minimal measures and nonsimplicial function cones.

[MATH-KMA 2008/267] Miroslav Bačák, Petr Hájek: Mazur intersection property for Asplund spaces.

[MATH-KMA 2008/268] Stanislav Hencl: On the weak differentiability of $u \circ f^{-1}$.

[MATH-KMA 2008/269] Amiran Gogatishvili, Luboš Pick, Jan Schneider: The rearrangement-invariant hull of a Besov space.

[MATH-KMA 2008/270] Ron Kerman, Luboš Pick: Explicit formulas for optimal rearrangementinvariant norms in Sobolev imbedding inequalities.

[MATH-KMA 2008/271] Petr Holický: Decompositions of Borel bimeasurable mappings between complete metric spaces.

[MATH-KMA 2008/272] Luboš Pick: Optimality of function spaces in Sobolev embeddings.

[MATH-KMA 2008/273] Jiř́ Spurný: Borel sets and functions in topological spaces.

[MATH-KMA 2008/274] Miroslav Kačena, Jiří Spurný: Affine Baire functions on Choquet simplices.

[MATH-KMA 2008/275] Jiří Jelínek, Dalibor Pražák: On the sign of Colombeau functions and applications to conservation laws.

[MATH-KMA 2008/276] Warren B. Moors, Jiří Spurný: On the topology of pointwise convergence on the boundaries of $L_{1}$-predual.

[MATH-KMA 2008/277] Hugo Beirão da Veiga, Petr Kaplický, Michael Růžička: Boundary regularity of shear thickening flows.

[MATH-KMA 2008/278] Petr Hájek, Gilles Lancien, Antonín Procházka: Weak ${ }^{*}$ dentability index of spaces $C([0, \alpha])$.

[MATH-KMA 2008/279] Robert Deville, Antonín Procházka: Parametric variational principle and residuality.

[MATH-KMA 2008/280] Michal Kraus: A note on the uniform approximation of continuous affine functions.

[MATH-KMA 2008/281] Tomáš Bárta: Smooth solutions of Volterra equations via semigroups.

[MATH-KMA 2008/282] Tomáš Bárta: Delayed quasilinear evolution equations with BV - coefficients.

[MATH-KMA 2008/283] Libor Veselý, Luděk Zajíček: On extensions of d.c. functions and convex functions.

[MATH-KMA 2008/284] Ondřej Kalenda, Wiesław Kubiś: The structure of Valdivia compact lines.

[MATH-KMA 2008/285] Petr Holický: Borel classes of uniformizations of sets with large sections.

[MATH-KMA 2008/286] Salvatore Leonardi, Jana Stará: Regularity results for the gradient of solutions of linear elliptic systems with VMO-coefficients and $L^{1, \lambda}$ data.

[MATH-KMA 2008/287] Andrea Cianchi, Luboš Pick: Optimal Gaussian Sobolev embeddings. 
[MATH-KMA 2008/288] Andrea Cianchi, Luboš Pick: An optimal endpoint trace embedding.

[MATH-KMA 2008/289] Robert Černý, Stanislav Hencl, Jan Kolár̆: Integral functionals that are continuous with respect to the weak topology on $W_{0}^{1, p}(\Omega)$.

[MATH-KMA 2008/290] Jaroslav Lukeš, Luboš Pick, Dušan Pokorný: On geometric properties of the spaces $L^{p(x)}$.

[MATH-KMA 2008/291] Miroslav Hušek, Antonio Pulgarín: Lattice-ordered groups of real continuous functions.

\section{9}

[MATH-KMA 2009/292] Petr Hájek, Antonín Procházka: $C^{k}$-smooth approximations of LUR norms.

[MATH-KMA 2009/293] Martin Koc: Upper porous sets in metric spaces which are not $\sigma$-lower porous.

[MATH-KMA 2009/294] Robert Černý, Silvie Mašková: On generalization of Mosers'theorem in the critical case.

[MATH-KMA 2009/295] Piotr Hajlasz, Jan Malý: On approximate differentiability of the maximal function.

[MATH-KMA 2009/296] Robert Černý, Silvie Mašková: A sharp form of an embedding into multiple exponential spaces.

[MATH-KMA 2009/297] W. Desch, E. Fašangová, J. Milota, G. Propst: Spectrum of a viscoelastic boundary damping problem.

[MATH-KMA 2009/298] W. Desch, E. Fašangová, J. Milota, G. Propst: Stabilization through viscoelastic boundary damping.

[MATH-KMA 2009/299] Jan Rataj, Luděk Zajíček: Properties of distance functions on convex surfaces.

[MATH-KMA 2009/300] Stanislav Hencl: Sharpness of the assumptions for the regularity of a homeomorphism.

[MATH-KMA 2009/301] Robert Černý: Integral functionals that are lower semicontinuous with respect to the weak topology on $W^{1, p}(\Omega)$.

[MATH-KMA 2009/302] Jakub Duda, Luděk Zajíček: On vector-valued curves that allow a $C^{1, \alpha}$ parametrization.

[MATH-KMA 2009/303] Jiř́ Spurný: Weights of boundaries of compact convex sets.

[MATH-KMA 2009/304] David A. Edwards, Ondřej Kalenda, Jiří Spurný: A note on intersections of simplices.

[MATH-KMA 2009/305] Martin Koc: On relations among metric derived numbers.

[MATH-KMA 2009/306] Domingo García, Ondřej Kalenda, Manuel Maestre: Envelopes of open sets and extending holomorphic functions on dual Banach spaces.

[MATH-KMA 2009/307] Stanislav Hencl, Jan Malý: Jacobians of Sobolev homeomorphisms.

[MATH-KMA 2009/308] Petr Holický, Roman Pol: On a question by Alexey Ostrovsky concerning preservation of completeness.

[MATH-KMA 2009/309] Lukáš Malý: Calderón-type theorems for operators of non-standard endpoint behavior on Lorentz spaces.

[MATH-KMA 2009/310] Petr Holický: Preservation of completeness by some continuous maps.

[MATH-KMA 2009/311] Luboš Pick: Weighted inequalities for integral and supremum operators.

[MATH-KMA 2009/312] Petr Holický: Descriptive classes of sets in nonseparable spaces.

[MATH-KMA 2009/313] Maurizio Grasselli, Dalibor Pražák, Giulio Schimperna: Attractors for nonlinear reaction-diffusion systems in unbounded domains via the method of short trajectories.

[MATH-KMA 2009/314] Antonio Avilés, Ondřej Kalenda: Compactness in Banach space theory selected problems.

[MATH-KMA 2009/315] Josef Daněček, Oldřich John, Jana Stará: Morrey space regularity for weak solutions of Stokes systems with VMO coefficients.

[MATH-KMA 2009/316] Dalibor Pražák, Kumbakonam R. Rajagopal: Mechanical oscillators described by a system of differential-algebraic equations. 